\title{
Experimental Demonstration of Mixed Formats and Bit Rates Signal Allocation for Spectrum-flexible Optical Networking
}

Borkowski, Robert; Karinou, Fotini; Angelou, Marianna; Arlunno, Valeria; Zibar, Darko; Klonidis, Dimitrios; Guerrero Gonzalez, Neil; Caballero Jambrina, Antonio; Tomkos, loannis; Monroy, Idelfonso Tafur

Published in:

OFC/NFOEC Technical Digest

Publication date:

2012

Document Version

Publisher's PDF, also known as Version of record

Link back to DTU Orbit

Citation $(A P A)$ :

Borkowski, R., Karinou, F., Angelou, M., Arlunno, V., Zibar, D., Klonidis, D., Guerrero Gonzalez, N., Caballero Jambrina, A., Tomkos, I., \& Monroy, I. T. (2012). Experimental Demonstration of Mixed Formats and Bit Rates Signal Allocation for Spectrum-flexible Optical Networking. In OFC/NFOEC Technical Digest (pp. OW3A.7). Optical Society of America.

\section{General rights}

Copyright and moral rights for the publications made accessible in the public portal are retained by the authors and/or other copyright owners and it is a condition of accessing publications that users recognise and abide by the legal requirements associated with these rights.

- Users may download and print one copy of any publication from the public portal for the purpose of private study or research.

- You may not further distribute the material or use it for any profit-making activity or commercial gain

- You may freely distribute the URL identifying the publication in the public portal 


\title{
Experimental Demonstration of Mixed Formats and Bit Rates Signal Allocation for Spectrum-flexible Optical Networking
}

\author{
Robert Borkowski ${ }^{1}$, Fotini Karinou ${ }^{1}$, Marianna Angelou ${ }^{2}$, Valeria Arlunno ${ }^{1}$, Darko Zibar ${ }^{1}$, Dimitrios Klonidis $^{2}$, \\ Neil Guerrero Gonzalez ${ }^{1}$, Antonio Caballero ${ }^{1}$, Ioannis Tomkos ${ }^{2}$, Idelfonso Tafur Monroy ${ }^{1}$ \\ ${ }^{1}$ DTU Fotonik, Dept. of Photonics Eng., Technical University of Denmark, Ørsteds Plads, Building 343, DK-2800 Kgs. Lyngby, Denmark \\ ${ }^{2}$ Athens Information Technology Center, Peania, Greece \\ rbor@fotonik.dtu.dk
}

\begin{abstract}
We report on an extensive experimental study for adaptive allocation of 16-QAM and QPSK signals inside spectrum flexible heterogeneous superchannel. Physical-layer performance parameters are extracted for use in resource allocation mechanisms of future flexible networks. OCIS codes: (060.4256) Networks, network optimization; (060.4510) Optical communications
\end{abstract}

\section{Introduction}

Spectrum-flexible solutions have recently attracted the interest of the research community due to their ability to allocate spectrum and capacity to incoming traffic demands in a dynamic fashion. Flexibility (i.e. bandwidth elasticity) is realized via adaptation in the wavelength, bit rate and modulation format that relies on advanced detection and transmission technologies. Coherent detection on one hand and multi-carrier orthogonal frequency division multiplexing (OFDM)-based transmission on the other, enable the grid-less networking paradigm for the optical transport of the next generation $[1,2]$. Bandwidth-variable transponders and optical cross-connects (OXCs) have been introduced to allow the efficient utilization of spectrum, allocating just the resources that are required [3]. Nonetheless, from the networking point of view, the introduction of flexibility imposes more complex requirements with respect to the spectrum and capacity allocation over the network link that stem from the fact that routing algorithms should now perform an exhaustive search through a large number of alternative solutions. This particularly applies in the operational phase of a network, where traffic is changing with time, leaving windows of spectrum of variable size unused [4]. Special routing algorithms are required to decide the route, the modulation format, the bandwidth and the location inside the available spectrum gaps for each incoming traffic demand. Interference from the neighboring channels has to be taken into account and sufficient guard bands ought to be considered. In order to achieve successful flexible spectrum allocation physical layer parameters such as available bandwidth, required optical signal-to-noise ratio (OSNR), number of subcarriers and required guard-band must be determined. So far there has been no experimental work on the requirement on the physical layer parameters for spectrum-flexible networking [1-6].

In this work, we explore the physical layer parameters for dynamic resource allocation in spectrum-flexible networks. We investigate the performance requirements needed for coherent optical OFDM signals with variable formats and bit rates to be established inside spectral windows of variable width. In order to study this concept we have set up an extensive experimental configuration to examine several networking scenarios in which different traffic demands are served by allocating various combinations of 14 Gbaud dual polarization (DP) 16-quadrature amplitude modulated (QAM) and DP quadrature phase-shift keyed (QPSK) signals within the available spectrum of a superchannel. The measured performance of these scenarios constitutes the required input that allows the network to adjust its characteristics with respect to offered capacity, spectral efficiency or reach.

\section{Concept and implemented scenarios}

In the flexible network, every connection between a pair of nodes consists of bands of spectrum with variable width (depending on the number of subcarriers and the requested traffic demand). During network operation the spectral occupancy changes dynamically with time leaving spectral gaps available for future demands [4]. We will refer to such spectral window as a Band-of-Interest (BOI). In a BOI, a source node can allocate a part or the entire band to a certain traffic demand. Depending on the traffic requirements, the adequate wavelength allocation, modulation format and bit rate should be used. This assignment should be determined by a cognitive subsystem, based on system performance knowledge acquired from past events and/or extrapolated from existing physical layer performance parameters [5].

To represent such events, we implemented a number of transmission scenarios for different BOI windows and wavelength allocation schemes, as well as for different modulation formats. Those scenarios are illustrated in the second column of Table 1. In all our cases, the optical spectrum consists of three bands. Two outer DP-QPSK bands, each one constituted by four subcarriers (red dashed arrows), surrounding the central band, i.e. the BOI (shadowed area). In scenarios A, B, C, and D two DP-16-QAM carriers with a variable spacing occupy the BOI. In scenarios G, $\mathrm{H}, \mathrm{F}$ and $\mathrm{E}$ a variable number of subcarriers of DP-QPSK occupy the BOI, see Table 1. We investigated BOI bandwidths of $98 \mathrm{GHz}$ (scenarios $\mathrm{A}, \mathrm{B}, \mathrm{G}, \mathrm{H}), 70 \mathrm{GHz}(\mathrm{C}, \mathrm{F})$ and $42 \mathrm{GHz}(\mathrm{D}, \mathrm{E})$. 


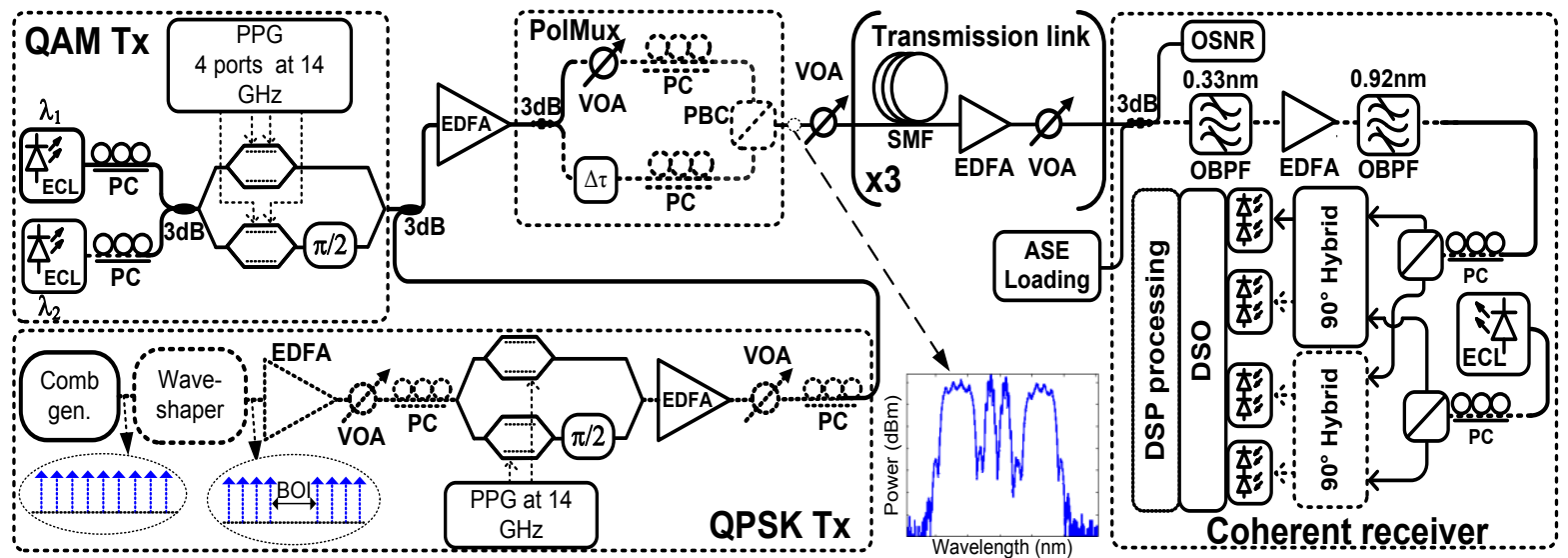

Fig. 1. Experimental setup.

\section{Experimental setup}

The experimental setup used for generation and detection of spectrum-flexible heterogeneous superchannel is shown in Fig. 1. We employ two independent transmitters for multi-carrier 16-QAM and QPSK optical signal generation. For a multi-carrier 16-QAM optical signal generation, we combine outputs of two independent external cavity lasers (ECLs) whose spectral separation can be varied. The combined output is then passed through an optical I/Q modulator which is driven by two 4-level electrical pulse amplitude modulated (PAM) signals at 14 Gbaud. For the generation of multi-carrier QPSK signal an optical comb is employed [1] and the spacing between the comb lines is fixed to $14 \mathrm{GHz}$. A spectrum-flexible superchannel is created using a WaveShaper to pass only two outer bands that surround the BOI, see Fig. 1. The output of the WaveShaper is then passed through an optical I/Q modulator which is driven by two binary electrical signals at 14 Gbaud in order to generate flexible multi-carrier QPSK channel. The spectrum-flexible heterogeneous superchannel is then generated by combining the multi-carrier 16-QAM and QPSK signals in an optical coupler. Next, the superchannel is amplified, polarization multiplexed (PolMux) and sent through a fiber link of standard single mode fiber (SMF) spans of lengths $68 \mathrm{~km}, 80 \mathrm{~km}$ and $104 \mathrm{~km}$. The launch power into each span of the $252 \mathrm{~km}$ long transmission link was fixed at $-2 \mathrm{dBm} / \mathrm{channel}$. At the coherent receiver, the heterogeneous superchannel is optically bandpass filtered, in order to filter out the signal in the BOI, and then applied together with a local oscillator (LO) to the input of a polarization diverse $90^{\circ}$ optical hybrid. The outputs of the hybrid are photodetected, sampled (40 GS/s) using digital sampling oscilloscope (DSO) and processed using offline digital signal processing (DSP) demodulation algorithms. By properly setting up the WaveShaper profile and activating/deactivating the QAM channels, all the combinations required for the studied scenarios were obtained. An example of the spectrum for scenario $A$ is shown as inset in Fig. 1. For scenarios E, F, G and $H$ the central wavelength was measured, while for scenarios A, B, C and D the carrier at the lower wavelength was measured.

\section{Measured results and discussion}

In order to evaluate the performance of the heterogeneous superchannel, bit error rate (BER) measurements are performed as a function of the OSNR $(0.1 \mathrm{~nm})$, for each of the flexible traffic allocation scenarios shown in Table 1. Moreover, we show in Table 1: a) the spectral width of the BOI; b) BOI capacity; c) total capacity of the superchannel; d) the achievable BOI spectral efficiency, defined as the ratio of the aggregated traffic within a BOI over the total spectral width of the available BOI; and d) the Required OSNR (ROSNR) in order to achieve BER of $1 \times 10^{-3}$ for the targeted channel in the BOI.

This study reveals that a $98 \mathrm{GHz}$ (7 subcarriers of $14 \mathrm{GHz}$ ) BOI can be filled with up to 7 subcarriers with DPQPSK data (scenario E) if ROSNR is above $22 \mathrm{~dB}$. By reducing the number of allocated DP-QPSK subcarriers within the BOI to 5 and 3 (scenarios $\mathrm{G}$ and $\mathrm{H}$ ) the ROSNR drops to $19.6 \mathrm{~dB}$ and $20.1 \mathrm{~dB}$ respectively, at an expense of reduced BOI spectral efficiency. It is conceivable that ROSNR levels can be interpolated for the cases of 2,4 and 6 DP-QPSK subcarriers since these are expected to lie within the ROSNR levels of the measured cases.

Alternatively, the $98 \mathrm{GHz}$ BOI can be filled with two DP-16-QAM subcarriers (scenario A) at a ROSNR level of at least $27.3 \mathrm{~dB}$. In this case, the DP-16-QAM subcarriers have been located $28 \mathrm{GHz}$ apart and at the centre of the BOI. Additional studies (scenario B) show that if the two DP-16-QAM subcarrier are located further apart to each other ( $56 \mathrm{GHz}$ spacing), but closer to the neighboring channels of the BOI (by $28 \mathrm{GHz}$ ) then the ROSNR increases to $30.9 \mathrm{~dB}$. This indicates that a traffic allocation mechanism should take into consideration not only the width of the BOI but also the spectral position of the channels within the BOI. 


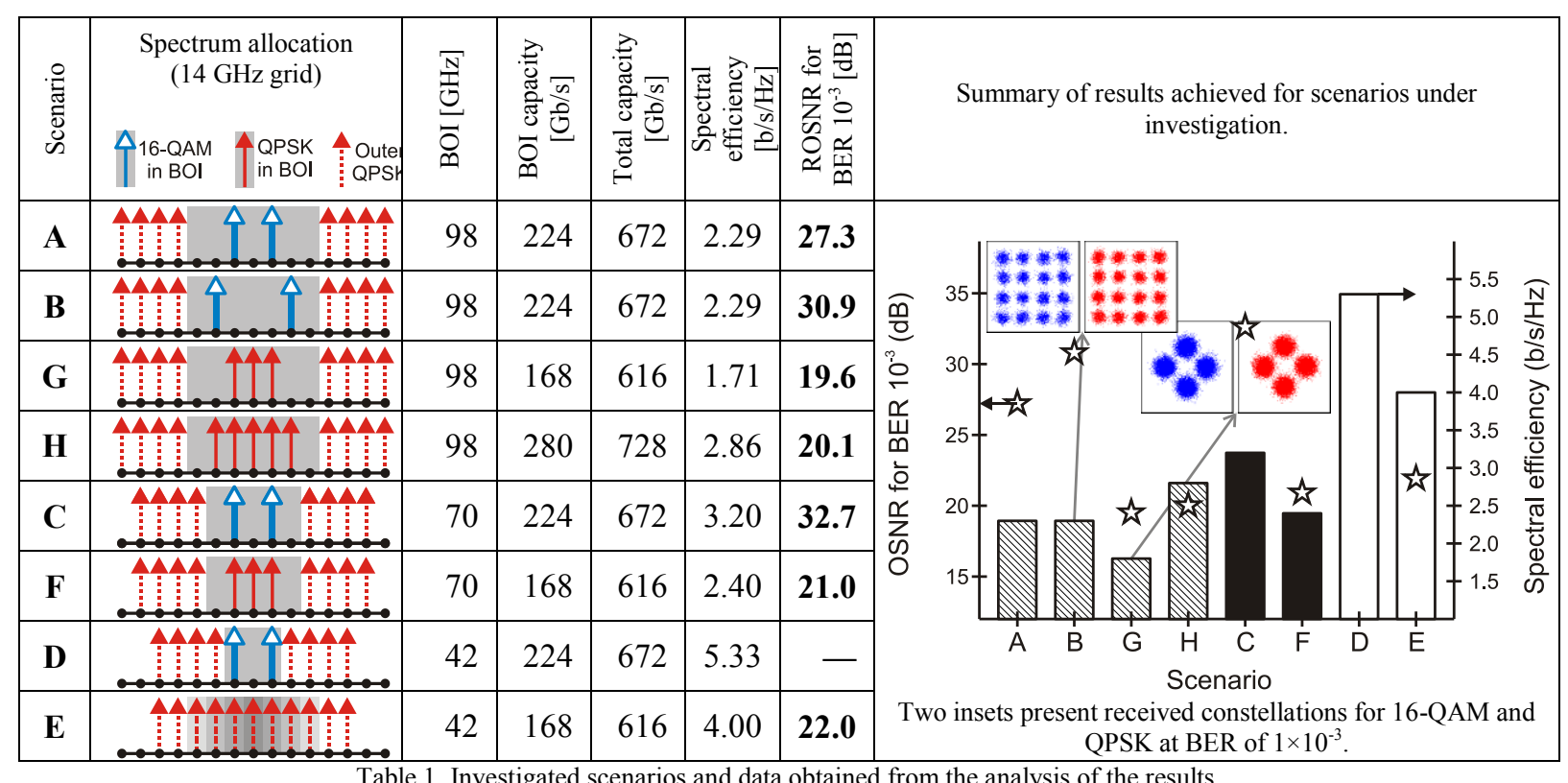

Considering a narrow BOI of $70 \mathrm{GHz}(5$ subcarriers of $14 \mathrm{GHz}$ ) being initially filled with 3 DP-QPSK subcarriers (scenario F), a ROSNR level of $21 \mathrm{~dB}$ is observed. This ROSNR level is similar to that of scenario H, denoting that the performance is mainly affected by the spectral distance of the allocated subcarriers from the neighboring channels of the BOI. The performance of the full DP-QPSK subcarrier allocation within the $70 \mathrm{GHz}$ BOI is defined again by scenario E (22 dB ROSNR). The same BOI of $70 \mathrm{GHz}$ can also be filled with 2 DP-16QAM subcarriers (scenario $\mathrm{C}$ ) resulting in an increased ROSNR level of $32.7 \mathrm{~dB}$ due to the close spectral proximity with the neighboring channels to the BOI.

Finally, it was observed that a narrow BOI of $42 \mathrm{GHz}$ could not accommodate two DP-16-QAM channels (scenario D) since the close spectral proximity $(14 \mathrm{GHz})$ to the non-orthogonal channels leads to increased errors. On the other hand the allocation of three DP-QPSK orthogonal channels is acceptable and refers to scenario E. A visual representation of the obtained results is shown in the figure in the last column of Table 1. ROSNR (stars) as well as spectral efficiency (bars) is compared. Insets in this figure show both polarizations of DP-16-QAM and DPQPSK at BER of $1 \times 10^{-3}$.

\section{Conclusions}

We have, for the first time, experimentally investigated the requirements on the physical layer parameters for a spectrum-flexible heterogeneous optical superchannel. We have identified the ROSNR levels for BER of $1 \times 10^{-3}$ for a number of scenarios with variable BOIs being dynamically filled with DP-QPSK and DP-16-QAM with variable bit rates. The importance of the result is that they are the basis for cognitive control algorithms to implement the new paradigm of heterogeneous reconfigurable optical networks.

\section{Acknowledgements}

We would like to thank LeCroy for providing WaveMaster DSOs, Finisar for the WaveShaper, ID Photonics for CoBrite DX1 lasers. We also thank Teit Poulsen from Altoo for his help. This work is partially supported by the European Community's FP7 projects CHRON under grant agreement no. 258644 and EUROFOS under grant agreement no. 224402.

\section{References}

[1] S. Chandrasekhar and L. Xiang, in Proc. ECOC 2010, paper Tu.3.C.5.

[2] T. Xia, et al., in Proc. OFC 2011, paper PDPA3.

[3] M. Jinno, et al., IEEE Commun. Mag., Vol. 47, Is. 11, 2009.

[4] K. Christodoulopoulos, et al., in Proc. OFC 2011, paper OTuI5.

[5] I. Tafur Monroy, et al., in Proc. ICTON 2011, paper Th.A1.1

[6] P. J. Winzer, et al., IEEE/OSA J. Lightwave Technol. 28, 547-556 (2010). 